\title{
СОВРЕМЕННАЯ НЕИНВАЗИВНАЯ ДИАГНОСТИКА ОСТРОГО АППЕНДИЦИТА У ВЗРОСЛЫХ
}

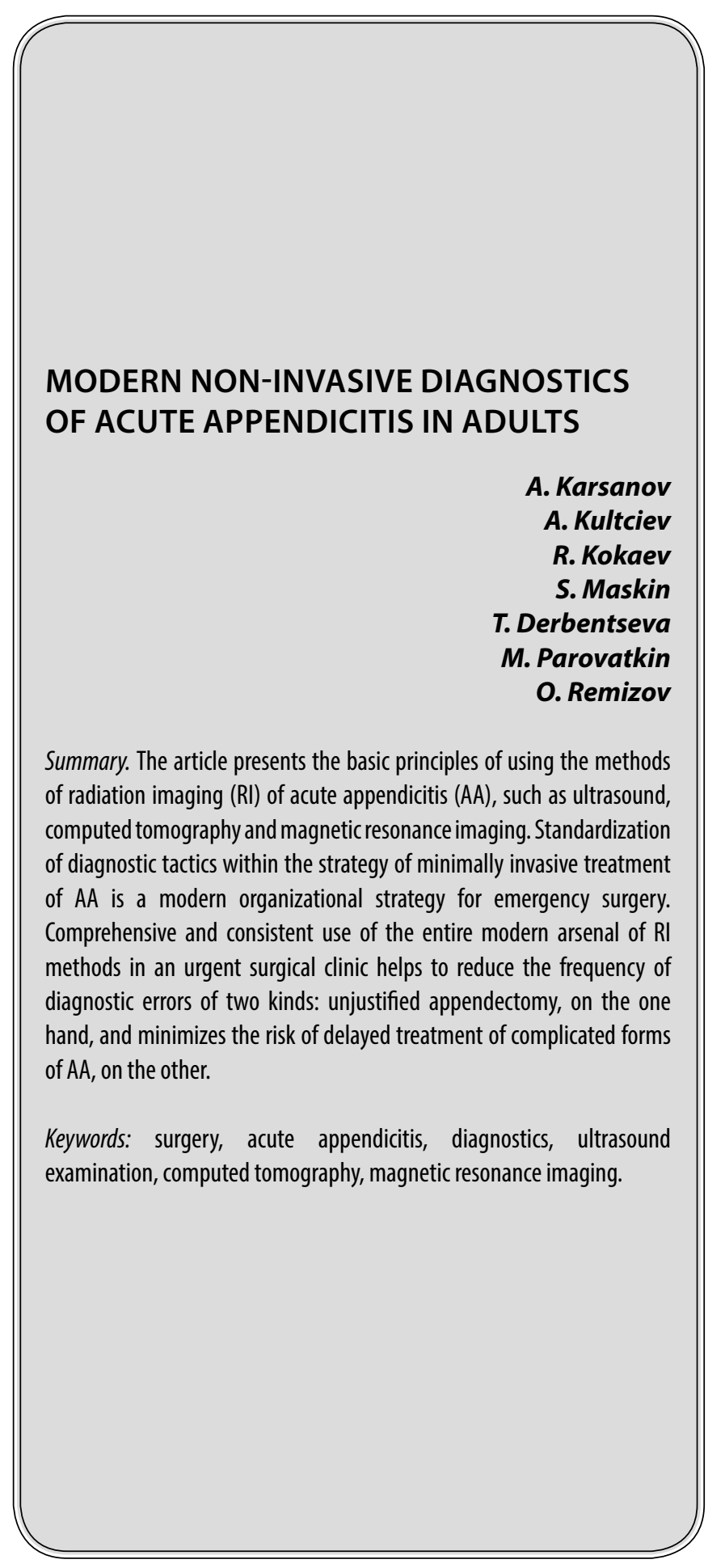

\author{
Карсанов Алан Мухарбекович \\ К.м.н., дочент, ФГБОУ ВО «Северо-Осетинская \\ государственная медицинская академия» \\ (2. Владикавказ) \\ karsan@inbox.ru \\ Кульчиев Ахсарбек Агубеевич \\ Д.м.Н., професссор, ФГБОУ ВО «Северо-Осетинская \\ государственная медицинская академия» \\ (2. Владикавказ) \\ kulchiev.ahsarbek@yandex.ru \\ Кокаев Роман Игоревич \\ ФГБОУ ВО «Северо-Осетинская государственная \\ медицинская академия» (2. Владикавказ) \\ sogma.rso@gmail.com \\ Маскин Сергей Сергеевич \\ Д.м.н., профессор, ФГБОУВО «Волгоградский \\ государственный \\ Медииинский университет» (2. Волгоград) \\ maskins@bk.ru \\ Дербенцева Татьяна Викторовна \\ К.м.Н., дочент, ФГБОУ ВО «Волгоградский \\ государственный медицинский университет» \\ (2. Волгоград) \\ TVDerbentseva@volgmed.ru \\ Пароваткин Михаил Иванович \\ К.м.Н., дочент, ФГБОУ ВО «Волгоградский \\ государственный \\ Медицинский университет» (2. Волгоград) \\ Ремизов Олег Валерьевич \\ Д.м.н., ректор, ФГБОУ ВО «Северо-Осетинская \\ государственная медицинская академия» \\ (2. Владикавказ) \\ oleg_remizov@mail.ru
}

Аннотация. В статье представлены базовые принципы использования методов лучевой визуализации (ЛВ) острого аппендицита (ОА), таких как ультразвуковое исследование, компьютерная и магнитно-резонансная томография. Стандартизация диагностической тактики в рамках стратегии малоинвазивного лечения ОА является современной организационной стратегией неотложной хирургии. Комплексное и последовательное использование всего современного арсенала методов ЛВ в ургентной хирургической клинике способствует снижению частоты диагностических ошибок двоякого рода: необоснованной аппендэктомии с одной стороны и минимизируют риск запоздалого лечения осложненных форм ОА с другой. 


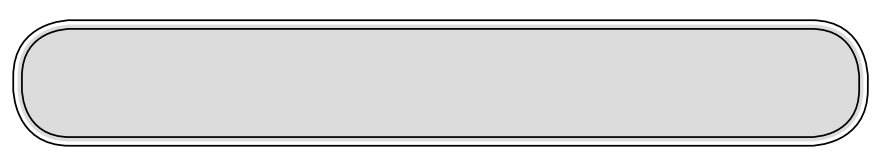

\section{Введение}

$\mathbf{T}$ акое собирательное понятие, как "острый живот" является неотложным угрожающим жизни пациентов состоянием и требует применения не столько детальной, сколько быстрой и тактически значимой диагностической программы [1, 2]. Одно из ведущих мест в общей структуре встречаемости острых хирургических заболеваний органов брюшной полости занимает острый аппендицит (ОА), вероятность развития которого в течение жизни прогнозируется примерно у $6,7 \%$ женщин и у 8,6\% мужчин [3-5].

Примерно от 6,5\% до 20\% пациентов с ОА имеют деструктивные и осложненные формы течения заболевания [6-8], в том числе и генерализованные внутрибрюшные инфекции и абдоминальный сепсис $[9,10]$. К современным трендам изучения концепции повышения качества и безопасности лечения пациентов с OA относится, в том числе, и поиск надежных принципов диагностики заболевания у беременных женщин и лиц пожилого возраста $[4,5,7,11,12]$.

\section{Стратегическое значение методов Аиагностики острого аппенАицита}

Говоря о стратегическом значении имеющихся в арсенале современного хирурга методов лучевой визуализации (ЛВ), следует обратиться к находящемуся в стадии утверждения и принятия в Российской Федерации проекту национальных клинических рекомендаций. Согласно им методы ЛВ в обследовании брюшной полости должны применяться после предварительной оценки вероятности ОА, проведенной по известным шкалам (Альварадо, AIR, AAS, RIPASA) [2, 13-15].

Ультразвуковое исследование (УЗИ) является обязательной опцией при констатации средней вероятности OA (по шкалам AIR, AAS) [2]. УЗИ - хорошее подспорье при проведении динамического мониторинга за пациентом, если первичный осмотр не подтвердил подозрения на ОА [7].

В клинической ситуации типичного болевого синдрома, когда безусловных ультразвуковых признаков острого воспаления червеобразного отростка (ЧО) не было выявлено или же в ситуации, когда последний не был идентифицирован вовсе, национальные эксперты рекомендуют выполнять низкодозную компьютерную томографию (КТ) брюшной полости $[2,4,16]$.
Ключевые слова: хирургия, острый аппендицит, диагностика, ультразвуковое исследование, компьютерная томография, магнитно-резонансная томография.

Здесь следует уточнить роль контрастного усиления компьютерного рентгеновского исследования. Большинство исследователей считают необходимым редукцию дозу лучевого воздействия сочетать с контрастированием при КТ исследовании $[2,4,16]$. Однако этот вопрос однозначно не разрешен, в первую очередь изза противоречий при оценке такой характеристики, как риск-польза предлагаемого метода.

Сторонников минимизации лучевого и иного воздействия при диагностики ОА должно обнадеживать то обстоятельство, что по итогам аналитического исследования М. Karul и соавт., КТ с низкой дозировкой лучевой нагрузки и без использования внутрисосудистого усиления оказалось столь же клинически эффективным, как и контрастированное КТ исследование со стандартной лучевой нагрузкой если при этом выявлялись как минимум пять КТ признаков ОА: утолщение стенки ЧО более 2 мм, диаметр поперечного сечения чО более 6 мм, наличие периаппендикулярного инфильтрата, наличие периаппендикулярного абсцесса, аппендиколит [17].

Высоко доказательной рекомендацией является то, что при отсутствии данных за ОА по результатам КТ пациентам с усиливающейся или сохраняющейся болью в правой подвздошной области рекомендуется выполнение диагностической лапароскопии для установки диагноза [2].

Кратко говоря о роли магнитно-резонансной томографии (МРТ) в диагностике ОА, то она имеет ограниченное применение у узкой категории пациентов, а именно у детей и беременных $[2,4,10,18,19]$. При подозрении на острый аппендицит у беременных пациенток последовательность применения методов ЛВ представлена УЗИ, а при его неинформативности - МРТ $[2,10,16,18$, $19]$.

\section{УЗИ в АИагностике острого аппенАицита}

Трансабдоминальное УЗИ является основным методом ЛВ для уточняющей диагностики ОА. УЗИ наиболее логически оправданно и повсеместно используется как продолжение клинического осмотра пациентов с острыми болями в брюшной полости и при констатации средней вероятности ОА [2, 5, 7, 14]. Метод доступен, воспроизводим в любом стационарном учреждении, пригоден для динамического использования, не требует подготов- 
ки пациента, не имеет противопоказаний. УзИ выполняется быстро и не доставляет дискомфорта пациенту.

Существенным недостатком УзИ является то, что при невизуализирующемся ЧО метод почти бесполезен, кроме его мониторинговых свойств. Помимо этого, было установлено, что доля неинформативных протоколов УЗИ при диагностике ОА у женщин значительно выше (38,5\%, против 6,2\% - у мужчин) [20]. Если впервые два триместра беременности УЗИ сопоставима по эффективности диагностики ОА с результатами обследования общей взрослой популяции, то в третьем триместре информативность метода резко снижается [17].

Оптимальным алгоритмом УЗИ при подозрении на ОА можно считать следующую последовательность. После полного обследования брюшной полости с помощью конвексного датчика (2-5 МГц) следует выполнить прицельную эхотомографию ЧО с использованием линейного датчика высокого разрешения (7,5-14 МГц). Исследователь ориентируется на боль пациента и ищет илеоцекальный угол. чО обычно можно визуализировать на медиальной стороне илеоцекального угла или, реже, в ретроцекальном положении. Нормальный отросток на продольном срезе выглядит как слепая трубчатая структура без перистальтики. Диаметр органа составляет менее 6 мм, а при инструментальном контакте датчиком аппендикс может уплощаться, что отражается на ультразвуковой картинке $[17,21]$.

Ультразвуковыми критериями ОА являются признаки воспалительных изменений в ЧО. Увеличение органа в размере, утолщение, неоднородность, инфильтрация (по гипоэхогенному типу) его стенки, наличие в просвете аппендиколита, наличие периаппендикулярной инфильтрации и абсцедирования, увеличенные единичные регионарные лимфатические узлы - все это эхотомографические признаки ОА [17].

Согласно сводной статистике М. Karul и соавт., чувствительность трансабдоминального УЗИ находится в пределах 71,2-92,0\%, в то время как специфичность - в диапазоне 83,3-96,0\% [17]. Комбинированное применение метода с трансабдоминальным УзИ повышает чувствительность до 97,3\%, а нижний порог достигнутой специфичности до $91 \%$.

С практической точки зрения крайне важно, что УзИ - это основной метод мониторирования динамики лечения аппендикулярного инфильтрата [26]. Метод вполне отчетливо позволяет документировать плотный воспалительный инфильтрат без жидкостных прослоек, образованный куполом слепой кишки, терминальным отделом подвздошной кишки, большим сальником и ЧО [22].
Современные принципы своевременности диагностики ОА сочетаются с таким свойством УЗИ, как навигация и минимальная инвазивность в лечении лечению аппендикулярных абсцессов. Следует напомнить, что при любых локализованных скоплениях гнойного экссудата УЗИ не только позволяет визуализировать полость альтерации, но и обеспечивает навигацию для пункционной санации и дренирования полости [23]. ЭХО-семиотика периаппендикулярного абсцесса давно известна и включает, в том числе, и визуализацию непосредственно полости жидкостного скопления.

По данным исследования, проведенного R. Sola и coавт., после принятия диагностического алгоритма, в котором УЗИ было приоритетным над КТ и включающего стандартизованные протоколы комбинированного ультрасонографического обследования, частота неопределенных результатов диагностики ОА снизилась с 44,3\% до $13,1 \%$, а положительные результаты у пациентов увеличились с $46,4 \%$ до $66,1 \%$ [24].

\section{КТ в Аиагностике острого аппенАицита}

Если анамнез, физикальное обследование, лабораторные маркеры воспаления (повышение С-реактивного белка и лейкоцитов) и трансабдоминальное УЗИ не позволили поставить диагноз ОА и беременность была исключена, необходимо выполнить КТ брюшной полости $[2,17,25]$. Это в первую очередь следует предпринять при невизуализированном во время ультразвукового исследования чО, что дает основания подозревать его атипичное расположение.

Результаты многочисленных метаанализов свидетельствуют об одном: в диагностике ОА разрешающая способность КТ превосходит УЗИ по критериям чувствительности (от $8 \%$ до 22\% по данным разных авторов) и специфичности (в среднем на 1-3\%) [2]. В поиске альтернативных диагнозов КТ-исследование демонстрирует чувствительность на уровне 84-88\% [4].

Наконец-то были получены данные, позволившие с оптимизмом смотреть на возможность снижения лучевой нагрузки на пациента. Финское одноцентровое рандомизированное исследование ОРТІСАР показало, что протокол низких доз с использованием внутривенного контрастирования не уступал стандартному протоколу КТ с точки зрения диагностической точности (79\% точных диагнозов при низких дозах и 80\% при стандартной КТ). Достоверность верификации осложненных морфологических форм ОА составила 79\% для обоих протоколов. Однако средняя доза облучения при КТ с низкой дозой была значительно ниже по сравнению со стандартной КТ (3,33 и 4,44 м3в соответственно) [26]. 
Основными информативными КТ признаками ОА являются: увеличении диаметра и поперечного размера чО более 6 мм, отсутствие визуализации его просвета, наличие неоднородной инфильтрации мезоаппендикса и экстралюминальное скопление жидкости и газа, что характерно для перфоративного ОА и абсцесса [7, 27].

Давно высказанное мнение, что при использовании только КТ пока не достигнут приемлемый уровень надежности в диагностике перфоративных форм ОА, пока не опровергнуто. Так по данным J. Debnath и соавт. [28], при последовательном применении УЗИ и КТ никакой дополнительной информации рентгеновского исследования не было получено, хотя уже при УзИ был визуализирован абсцесс в зоне исследования.

\section{МРТ в $\triangle$ иагностике острого аппенАицита}

Международные согласительные документы рекомендуют МРТ в качестве метода визуализации второй линии в неубедительных случаях после УзИ, хотя МРТ может использоваться как метод визуализации первой линии, если этот метод в клинике доступен [29].

Выше было сказано о возросшей значимости УзИ В диагностике ОА, однако это не распространяется на популяцию беременных женщин со сроком позднее первого триместра. У этой категории хирургических пациентов основным недостатком УзИ является высокая частота невизуализируемости ЧО, которая составляет от $34,1 \%$ до $71 \%$ при подтвержденных в последствие острых воспалительных изменениях в органе интереса [30].

Другая крайность при тактике изолированного использования УзИ подозрении на ОА у беременных на средних и поздних сроках заключатся в том, что до $30 \%$ выполненных аппендэктомий в подобной клинической ситуации оказываются необоснованными, а частота ложноотрицательных заключений при УзИ беременных, опровергнутых после МРТ, достигает 8\% [18].

Согласно результатам 3 метаанализов, опубликованных за последние 10 лет, МРТ при ОА на фоне беременности имеет чувствительность - 90,5\%, 94\% и 91,8\%, специфичность - 98,6\%, 97\% и 97,9\%, положительную прогностическую ценность - 86,3\% и отрицательную прогностическую ценность - 99,0\% [2]. Частота невизуализируемости ЧО оказалась наивысшая в 3 триместре, когда наибольшая степень анатомического искажения возникает из-за беременной матки, что, однако, не мешает рассматривать МРТ как современный стандарт неинвазивной диагностики ОА у беременных.

\section{Зак^ючение}

Одним из основополагающих принципов научного подхода к повышению качества лечения и безопасности пациентов в ургентной хирургии является использования общедоступных, воспроизводимых и высоко результативных методов своевременной диагностики, что позволит быстро и в полном объеме установить диагноз, определить показания к оперативному лечению, а также использовать минимально инвазивные хирургические технологии.

Существенное место в регламентирующих клиническую деятельность рекомендациях отводится стандартизации диагностики и алгоритмированию тактики у пациентов особо высокого риска неудовлетворительных результатов. Применительно к ОА к таковым относятся беременные женщины, дети, пациенты пожилого и старческого возраста, а также лица со сложным коморбидным статусом, у которых ОА ожидаемо протекает атипично.

Именно в силу указанных обстоятельств перед хирургическим сообществом, как и прежде, стоит задача по научному анализу и систематизации современных данных, синтезу жизнеспособных концепций и интегрированию в клиническую практику лучших моделей комплексной диагностики ОА.

Компонентами неинвазивной лучевой визуализации, используемыми в мировой хирургической практике для диагностики ОА являются: УЗИ, КТ, МРТ. Набор не широк, но от правильной и логически оправданной последовательности применения этих методов зависит успех дооперационной верификации деструктивных и осложненных форм ОА в ситуации, относящейся к т.н. средневероятностному клиническому случаю.

Когда клиническая картина ОА очевидна и риски оперативного вмешательства минимальны или оправданы, то достаточно констатировать клинические либо ультрасонографические признаки заболевания червеобразного отростка. При малой вероятности аппендикулярного генеза болевого синдрома либо когда боли купированы, УзИ используется в качестве метода дополнительного исключение острого воспаления в ЧО.

Таким образом, использование современных визуализационных методов (УЗИ, КТ, МРТ) является необходимым условием, повышающим информативность базовой клинико-лабораторной диагностики ОА в сложных клинических случаях и у проблемных категорий пациентов.

Диагностическая и клиническая ценность всех методов визуализационной диагностики различна, но она повышается при логичном и последовательно выверенном использовании хирургами всего имеющегося в клинике арсенала 
средств дооперационной верификации повышается при их комплексном и последовательном применении.

Важно сохранять понимание, что предполагаемая диагностическая ценность метода не должна быть до- минантой перед безусловным риском технологического вреда для здоровья пациента, как, например, в случае с полнодозной КТ, а соблюдать разумный баланс и использовать весь арсенал диагностических методов в интересах каждого пациента.

\section{ЛИТЕРАТУРА}

1. Острый аппендицит / А.В. Сажин, Т.В. Нечай, А.И. Кириенко.— Москва: 000 «Медицинское информационное агентство», 2019. — 208 c.

2. Di Saverio S., Podda M., De Simone B. et. al. Diagnosis and treatment of acute appendicitis: 2020 update of the WSES Jerusalem guidelines // World J. Emerg Surg. 2020.—№ 1.—P. 27.

3. Ревишвили А.Ш., Сажин В.П., Оловянный В.Е., Захарова М.А. Современные тенденции в неотложной абдоминальной хирургии в Российской Федерации // Хирургия. Журнал им. Н.И. Пирогова. — 2020.— № 7.-С. 6-11.

4. Fugazzola P., Ceresoli M., Agnoletti V. at al. The SIFIPAC/WSES/SICG/SIMEU guidelines for diagnosis and treatment of acute appendicitis in the elderly (2019 edition) // World J. Emerg Surg. — 2020.— № 1.—P. 19.

5. Monsonis B., Mandoul C., Millet I., Taourel P. Imaging of appendicitis: Tips and tricks // Eur. J. Radiol. — 2020 — Vol. 130:109165. doi: 10.1016/j.ejrad.2020.109165.

6. Маскин С.С., Карсанов А.М., Дербенцева Т.В., Матюхин В.В., Карсанова 3.0. Дифференцированный выбор тактических решений при генерализованной внутрибрюшной инфекции // Московский хирургический журнал. - 2015.— № 1.—C. 36-40.

7. Mostbeck G., Adam E.J., Nielsen M.B. et al. How to diagnose acute appendicitis: ultrasound first // Insights Imaging. — 2016. — № 2.— P. 255 -263.

8. Карсанов А.М., Кульчиев А.А., Караев Т.Р., Кокаев И.П., Вахоцкий В.В. Роль современных методов лучевой визуализации при внутрибрюшных гнойно-воспалительных осложнениях толстокишечного генеза // Хирургия. Журнал им. Н.И. Пирогова. - 2015.— № 5.— С. 75-79.

9. Карсанов А.М., Ремизов 0.В., Маскин С.С., Кульчиев А.А., Карсанова 3.0. Диагностика сепсиса // Вестник хирургии.— 2016.— № 6.—C. 98-103.

10. Карсанов А.М., Маскин С.С., Слепушкин В.Д., Карсанова 3.0., Дербенцева Т.В., Саламова Ф.Т., Караев Т.Р. Клинико-эпидемиологическое значение системного воспаления и сепсиса // Вестник хирургии. - 2015.— № 4.—C. 99-103.

11. Сажин А.В., Курцер М.А., Коноплянников А.Г., Ивахов Г.Б., Панин А.В., Сон Д.А., Шуляк Г.Д., Серебренникова Ю.А. Осложненные формы острого аппендицита у беременных // Хирургия. Журнал им. Н.И. Пирогова.— 2019.— № 4. — С. $15-23$.

12. Salminen P., Paajanen H., Rautio T. et al. Antibiotic therapy vs appendectomy for treatment of uncomplicated acute appendicitis: the APPAC randomized clinical trial // JAMA. - 2015. — № 23. - P. 2340-2348.

13. Andersson M., Kolodziej B., Andersson R.E. et al. Randomized clinical trial of appendicitis inflammatory response score-based management of patients with suspected appendicitis // Br.J. Surg.— 2017.— № 11.—P. 1451-1461.

14. Scott A.J., Mason S.E., Arunakirinathan M. et al. Risk stratification by the Appendicitis Inflammatory Response score to guide decision-making in patients with suspected appendicitis // Br.J. Surg.— 2015.— № 5.—P. 563-572.

15. Sammalkorpi H.E., Mentula P., Savolainen H. et al. The introduction of Adult Appendicitis Score reduced negative appendectomy rate // Scand J. Surg. — 2017. № 3.- - P. 196-201.

16. «0стрый аппендицит у взрослых» Клинические рекомендации Российского общества хирургов. 2020. Электронный ресурс: http://www.xn-— 9sbdbejx7bdduahou3a5d.xn — p1ai/stranica-pravlenija/klinicheskie-rekomendaci/urgentnaja-abdominalnaja-hirurgija/-ostryi-apendicit-u-vzroslyh-2020.html.

17. Karul M., Berliner C., Keller S., Tsui T.Y., Yamamura J. Imaging of appendicitis in adults // Rofo. — 2014. — № 6. — P. 551-558.

18. Amitai M.M., Katorza E., Guranda L. et al. Role of emergency magnetic resonance imaging for the workup of suspected appendicitis in pregnant women // Isr. Med. Assoc. J.— 2016. — № 10.—P. 600-604.

19. Patel D., Fingard J., Winters S. et al. Clinical use of MRI for the evaluation of acute appendicitis during pregnancy // Abdom. Radiol.— 2017.— № 7.— P. 18571863.

20. Rosenkrantz A.B., Labib A., Ginocchio L.A., Babb J.S. Evaluation for suspected acute appendicitis in the emergency department setting: a comparison of outcomes among three imaging pathways // Clin. Imaging.—2016.— № 4.—P. 788-792.

21. Binkovitz L.A., Unsdorfer K.M., Thapa P. et al. Pediatric appendiceal ultrasound: accuracy, determinacy and clinical outcomes // Pediatr. Radiol.— 2015.— № 13.— P. 1934-1944.

22. Epifanio M., de Medeiros Lima M.A., Corrêa P., Baldisserotto M. An imaging diagnostic protocol in children with clinically suspected acute appendicitis // Am. Surg.2016.—№ 5.—P. 390-396.

23. Blumfield E., Nayak G., Srinivasan R. et al. Ultrasound for differentiation between perforated and nonperforated appendicitis in pediatric patients // Am.J. Roentgenol.— 2013.— № 5.—P. 957-962.

24. Sola R., Theut S.B., Sinclair K.A. Rivard D.C. et al. Standardized reporting of appendicitis-related findings improves reliability of ultrasound in diagnosing appendicitis in children // Pediatr. Surg.— 2018.— № 5.—P. 984-987.

25. Remizov 0.V., Sazhin V.P., Karsanov A.M. On bioethical component of patient safety in surgery // Bioethics. — 2017.— № 1.— P. 44-48.

26. Sippola S., Virtanen J., Tammilehto V. et al. The accuracy of low-dose computed tomography protocol in patients with suspected acute appendicitis: the OPTICAP study // Ann. Sur.— 2020.— № 2.—P. 332-338. 
27. Hong H.S., Cho H.S., Woo J.Y. et al. Intra-appendiceal air at CT: is it a useful or a confusing sign for the diagnosis of acute appendicitis? // Korean J. Radiol.— 2016. № 1. - P. 39-46.

28. Debnath J., Sharma V., Ravikumar R. et al. Clinical mimics of acute appendicitis: is there any role of imaging? // Med. J. Armed. Forces India. — 2016.— № 3.— P. 285-292.

29. Garcia E.M., Camacho M.A, Karolyi D.R. et al. ACR appropriateness criteria ${ }^{\circledR}$ right lower quadrant pain-suspected appendicitis // J. Am. Coll. Radiol.— 2018.№ 11S. - P. 373-387.

30. Segev L., Segev Y., Rayman S. et al. The diagnostic performance of ultrasound for acute appendicitis in pregnant and young nonpregnant women: A case-control study // Int. J. Surg. — 2016.— № 34.—P. 81-85.

( ) Карсанов Алан Мухарбекович ( karsan@inbox.ru ), Кульчиев Ахсарбек Агубеевич ( kulchiev.ahsarbek@yandex.ru ),

Кокаев Роман Игоревич ( sogma.rso@gmail.com ), Маскин Сергей Сергеевич ( maskins@bk.ru ),

Дербенцева Татьяна Викторовна (TVDerbentseva@volgmed.ru),

Пароваткин Михаил Иванович, Ремизов Олег Валерьевич ( oleg_remizov@mail.ru).

Журнал «Современная наука: актуальные проблемы теории и практики»

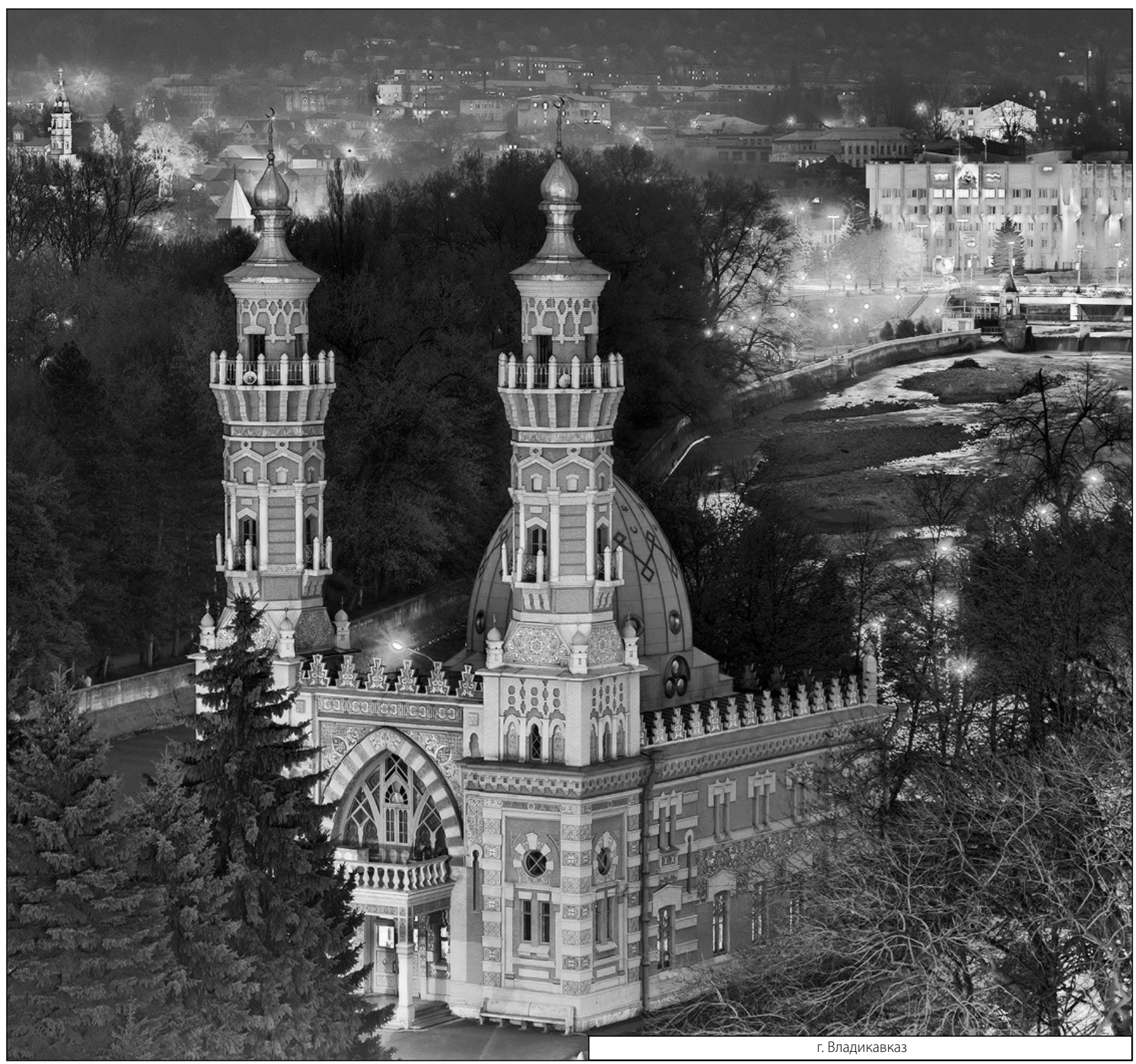

